\title{
Courbes d'ébullition en convection forcée. Première partie : Revue bibliographique
}

\author{
Boiling curves with forced convection \\ Part 1 : Bibliographical review
}

\author{
M. Llory* \\ D. Gentile** \\ * EDF, Direction des Etudes et Recherches, 78400 Chatou \\ ** ENSTA, Groupe Phénomènes d'interface, 91120 Palaiseau
}

M. Afify**

\begin{abstract}
Les propriétés de transfert thermique entre une paroi chauffée et un écoulement de liquide en ébullition sont présentées globalement à l'aide du concept de courbe d'ébullition et de stabilité thermique. Dans la première partie, on introduit ce concept en le plaşant dans le cadre des principales applications industrielles et des codes de calcul utilisés pour la süreté nucléaire. Une revue bibliographique fait le point de létat des connaissances et montre l'intérêt de cette notion qui tend à se généraliser depuis une décennie. Compte tenu de l'existence d'instabilités thermiques dans les mécanismes d'ébullition. l'obtention de la courbe ne peut être réalisée que par des dispositifs expérimentaux appropriés. Une brève description de ces différents dispositifs est présentée. On insiste plus particulièrement sur la stabilisation électronique (asservissement) en liaison avec le concept de stabilité thermique. Ce concept permet de déduire un critère de stabilité, condition nécessaire à la réalisation du dispositif expérimental assurant la stabilité dynamique du processus d'ébullition.
\end{abstract}

The properties of heat transfer between a heated wall and a boiling fluid flow are described generally; using the concept of boiling curve and thermal stability. In Part l, this concept is introduced, placing it in the framework of the main industrial applications and the computation codes used for nuclear safety: A bibliographical review sums up the state of knowledge and shows the interest of this concept. which is tending to become generalized over the last decade. Considering the existence of thermal instabilities in boiling mechanisms, the only way to obtain the curve is by appropriate experimental devices. $A$ brief description of these devices is given. The emphasis is placed on electronic stabilization (feedback) in connection with the concept of thermal stability. This concept allows determination of a stability criterion, a requisite for designing an experimental device ensuring dynamic stability of the boiling process.

\section{Introduction - Généralités - Revue bibliographique}

On se place principalement, dans cet article, dans le cas des écoulements de fluides en ébullition le long de surfaces chauffantes cylindriques verticales, telles que tubes ou barreaux chauffants, dans des sections de forme circulaire ou annulaire. Dans ces conditions, les écoulements sont supposés axisymétriques et monodimensionnels. Ce cas correspond, de loin, aux expériences les plus fréquentes effectuées en ébullition.

\subsection{Généralités}

Les propriétés locales de transfert thermique, entre une paroi chauffante et un liquide en ébullition, sont habituellement représentées par une loi de comportement reliant la densité de flux thermique locale échangée $q$ à l'écart de température $\theta$ entre la paroi en contact avec le liquide en ébullition $T_{p}$ et le liquide lui-même $T_{f}\left(\theta=T_{p}-T_{f}\right)$. On peut également faire intervenir le coefficient d'échange thermique qui est le rapport de ces deux grandeurs : $h=q / \theta$.

L'ébullition se produit au contact d'une paroi chauffante, soit lorsque le liquide a atteint l'état de saturation $\left(T_{f}=T_{\text {sat }}\right)$ : c'est le cas de l'ébullition saturée; soit lorsque le fluide étant à l'état liquide $\left(T_{f}<T_{\text {sat }}\right)$, la température de paroi est supérieure à la température de saturation du fluide correspondant à sa pression locale $\left(T_{p}>T_{\text {sat }}(P)\right)$ : c'est le cas de l'ébullition sous-saturée.

Il existe, par ailleurs, deux grands modes d'ébullition : - l'ébullition stagnante ou en convection naturelle ("pool boiling"): dans laquelle la surface chauffante est immergée dans un bain de liquide stagnant;

- l'ébullition en convection forcée ("forced convection boiling ") dans laquelle le liquide s'écoule le long de la surface chauffante. 


\section{Notations}

A Aire de l'interface paroi cylindrique - fluide bouillant

$B_{0}, B$ Pôles des fonctions de transfert du premier ordre

$$
\frac{\delta \theta(s)}{\delta q_{e}(s)} \text { et } \frac{\delta \theta(s)}{\delta i(s)} \text { respectivement }
$$

$C_{m} \quad$ Chaleur massique à pression constante de la paroi chauffante

(J.kg $\left.{ }^{-1} \cdot \mathrm{K}^{-1}\right)$

$C(s) \quad$ Fonction de transfert du filtre correcteur

$d \quad$ Diamètre équivalent

(m)

$d_{i}, d_{c} \quad$ Diamètres intérieur et extérieur d'un tube (m)

$D \quad$ Dimension caractéristique de la paroi cylindrique :

$$
\frac{\text { volume }}{\text { aire de l'interface }}=\frac{V}{A}
$$

$e \quad$ Epaisseur de la paroi chauffante

$G \quad$ Flux massique $\left(\mathrm{kg} \cdot \mathrm{m}^{-2} \cdot \mathrm{S}^{-1}\right)$ ou gain du système en boucle fermée

$h \quad$ Coefficient de transfert thermique $\left(\mathrm{W} \cdot \mathrm{m}^{-2} \cdot \mathrm{K}^{-1}\right)$

$h_{0} \quad$ Coefficient de transfert thermique du fluide

chauffant à la paroi $\left(\mathrm{W} \cdot \mathrm{m}^{-2} \cdot \mathrm{K}^{-1}\right)$

$H \quad$ Enthalpie massique $\quad\left(\mathrm{J}_{\mathrm{kg}}^{-1}\right)$

$H_{t} \quad$ Enthalpie massique du liquide saturé $\quad\left(\mathrm{J} \mathrm{kg}^{-1}\right)$

$i \quad$ Intensité du courant

$K_{0}, K$ Termes des fonctions de transfert du premier ordre

$$
\frac{\delta \theta(s)}{\delta q_{c}(s)} \text { et } \frac{\delta \theta(s)}{\delta i(s)} \text { respectivement }
$$

$\ell \quad$ Longueur chauffante amont

$L \quad$ Chaleur latente de vaporisation

$P \quad$ Pression moyenne de l'écoulement dans une section $q \quad$ Densite de flux thermique (W. $\mathrm{m}^{-2}$ )

$q_{i}, q_{c} \quad$ Densité de flux thermique rapportée au système interne (le fluide en ébullition), au système externe (la source de chaleur, y compris la paroi chauffante)

(W. $\mathrm{m}^{-2}$ )

$R \quad$ Résistance électrique

$s \quad$ Variable de la transformation de Laplace

$T_{c} \quad$ Température moyenne locale du fluide chauffant

$T_{f} \quad$ Température moyenne du fluide en ébullition $\left({ }^{\circ} \mathrm{C}\right)$

$\begin{array}{lll}T_{p} & \text { Température de la paroi chauffante } \quad\left({ }^{\circ} \mathrm{C}\right)\end{array}$

$\begin{array}{lll}T_{\text {sat }} & \text { Température de saturation } & \left({ }^{\circ} \mathrm{C}\right)\end{array}$

$V \quad$ Volume de la paroi cylindrique chauffante $\left(\mathrm{m}^{3}\right)$

$x \quad$ Titre massique à l'équilibre thermodynamique

$\alpha \quad$ Coefficient de variation de la résistivité électrique en fonction de la température $\left(\Omega . \mathrm{K}^{-1}\right)$ Ecart entre la consigne et le signal

Conductivité thermique

(W. $\left.\mathrm{m}^{-1} \cdot \mathrm{K}^{-1}\right)$

$\lambda$

$\rho_{m}$

$\theta$ masse volumique de la paroi chauffante $\left(\mathrm{kg} \cdot \mathrm{m}^{-3}\right)$ Ecart de température entre la paroi chauffante et le liquide en ébullition : $T_{p}-T_{f}$ ou $T_{p}-T_{\mathrm{sat}}\left({ }^{\circ} \mathrm{C}\right)$

\section{Indices}

$\delta \quad$ Ecart par rapport à une valeur stationnaire

c Maximum de la courbe d'ébullition (conditions critiques)

$m$ Minimum de la courbe d'ébullition (point de LEIDENFROST).

Le premier mode d'ébullition correspond au fonctionnement des chaudières à convection naturelle. Dans certaines circonstances accidentelles, l'ébullition stagnante peut se rencontrer momentanément dans des systèmes qui, habituellement, sont le siège d'ébullition en convection forcée ou, au moins, d'écoulements forcés sous-saturés : c'est le cas dans les réacteurs à eau sous pression lorsque l'apparition accidentelle d'une brèche dans le circuit primaire provoque la dépressurisation de celui-ci. $\mathrm{Ce}$ mode d'ébullition a fait l'objet et fait encore l'objet d'un très grand nombre d'investigations expérimentales, notamment en raison de sa plus grande simplicité, par rapport à l'ébullition en convection forcée et de sa plus grande facilité de mise en œuvre [1], [2], [3].

Le deuxième mode d'ébullition correspond au fonctionnement des chaudières et générateurs de vapeur à circulation forcée, notamment, de l'industrie nucléaire : générateurs de vapeur chauffés au sodium de la filière à neutrons rapides, à l'hélium, pour la filière à haute température HTR, par exemple. Il peut se rencontrer dans des circonstances accidentelles, comme on l'a évoqué au paragraphe précédent. Ce deuxième mode d'ébullition est plus complexe que le premier, en raison du plus grand nombre de paramètres ayant une influence sur les propriétés de transfert thermique et sur l'écoulement. Il a, cependant, fait l'objet d'un très grand nombre d'études, tant théoriques qu'expérimentales.

Il apparaît intéressant de rappeler que le nombre d'articles traitant des écoulements diphasiques et de l'ébullition croissait, dans les années 1970, de façon exponentielle. En 1960, on recensait environ 3000 références disponibles dans le domaine. L'extrapolation donne un total de près de 10000 références en 1970 [4]. En 1985, la même extrapolation donne un total d'environ 40000 références. Ces chiffres donnent une idée de l'importance des travaux effectués, sous l'impulsion principalement de 


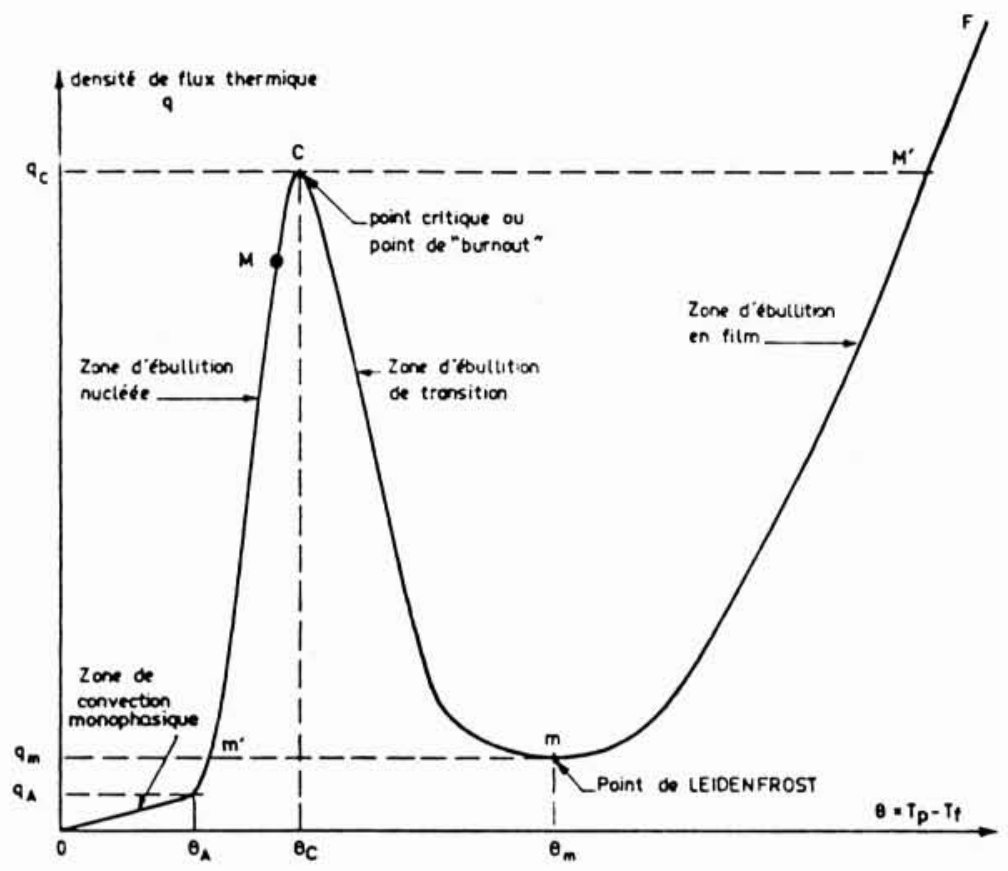

Figure 1. - Allure d'une courbe d'ébullition.

l'industrie nucléaire et, à un degré moindre, des industries aéronautiques et spatiales.

On distingue, par ailleurs, deux types de systèmes en ébullition, suivant le mode de chauffe appliqué [5] :

- les systèmes "à flux imposé " : c'est la densité de flux thermique qui est imposée et est considérée comme une variable indépendante. C'est le cas des systèmes chauffés électriquement ou des éléments combustibles d'un réacteur nucléaire. C'est ce qui explique, en dehors d'une certaine facilité de mise en œuvre, que la plupart des expériences réalisées en ébullition l'aient été en utilisant le chauffage électrique:

- les systèmes "à température imposée " : c'est la température de paroi qui est imposée et est considérée comme la variable indépendante. Ce cas correspond approximativement au fonctionnement des appareils du type " échangeurs de chaleur». L'apport de chaleur s'effectue par un autre fluide (par exemple, un fluide en condensation).

Cette distinction correspond, toutefois, à des systèmes " idéaux ". On verra, au chapitre suivant, qu'un système dissipant de l'énergie par effet Joule, n'est pas en toute rigueur un système à "flux imposé " et qu'un système utilisant un fluide chauffant, n'est pas, dans tous les cas, un système à "température imposée ".

\subsection{Courbes d'ébullition en convection forcée}

L'hypothèse des " conditions locales " conduit à supposer que les propriétés de transfert thermique locales entre la paroi chauffante et le liquide en ébullition peuvent être déterminées uniquement à partir des valeurs locales des paramètres thermohydrauliques, des caractéristiques géométriques et des conditions locales d'état de surface.

Avec cette hypothèse, la densité de flux thermique locale échangée est une fonction de l'écart de température $\theta=T_{n}-T_{f}$, de la pression $P$, du flux massique $G$, de l'enthalpie locale $H$ et des caractéristiques géométriques (diamètre $d$ du tube chauffant) et d'état de surface. Si l'on néglige, dans un premier temps, l'influence de l'état de surface, on peut poser :

$$
q=q(\theta, P, G, H, d) .
$$

Pour un liquide saturé, on introduit le plus souvent le titre massique à l'équilibre thermodynamique :

$$
x=\frac{H-H_{t}}{L}
$$

Pour un diamètre de tube imposé et lorsque $P$ et $G$ sont fixés, en particulier, on a donc pour un liquide saturé :

$$
q=q(\theta, x)
$$

Le transfert thermique est représenté par une famille d'isotitres. Une isotitre particulière, notamment à faible titre, a, en général, l'allure de la figure $I$, c'est-à-dire une allure en " $\mathrm{S}$ ». On peut y distinguer les trois principaux régimes d'ébullition :

- ébullition nucléée et vaporisation en convection forcée pour $\theta<\theta_{c}$;

- ébullition de transition pour $\theta_{c}<\theta<\theta_{m}$. Ce régime est caractérisé par le fait que la densité de flux thermique décroît lorsque $\theta$ croît : $\partial q / \partial \theta<0$;

- ébullition en film pour $\theta>\theta_{m}$. 


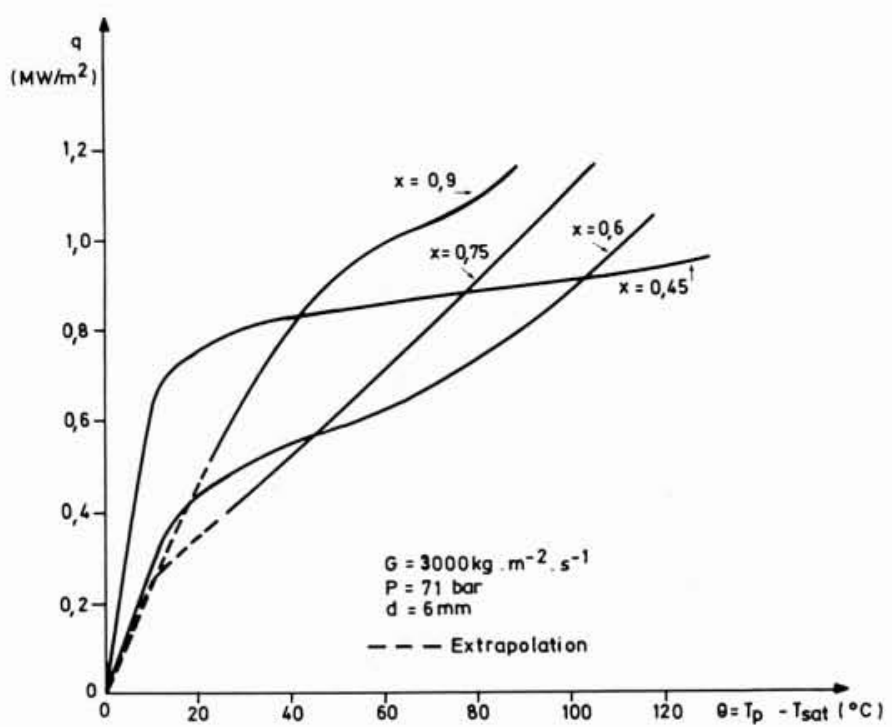

Figure 2. - Courbes d'ébullition monotones croissantes de l'eau. Effet du titre massique local. (Courbes tirées des données expérimentales d'Era [54]).

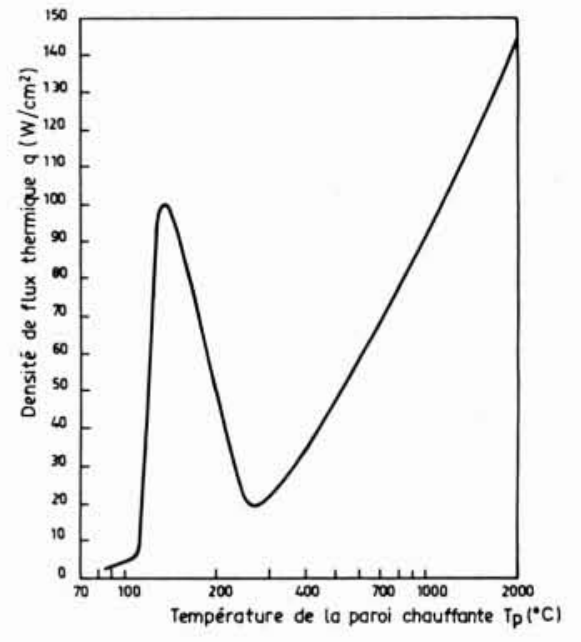

Figure 3. - Courbe d'ébullition stagnante de l'eau à la pression atmosphérique, d'après Collier [20].

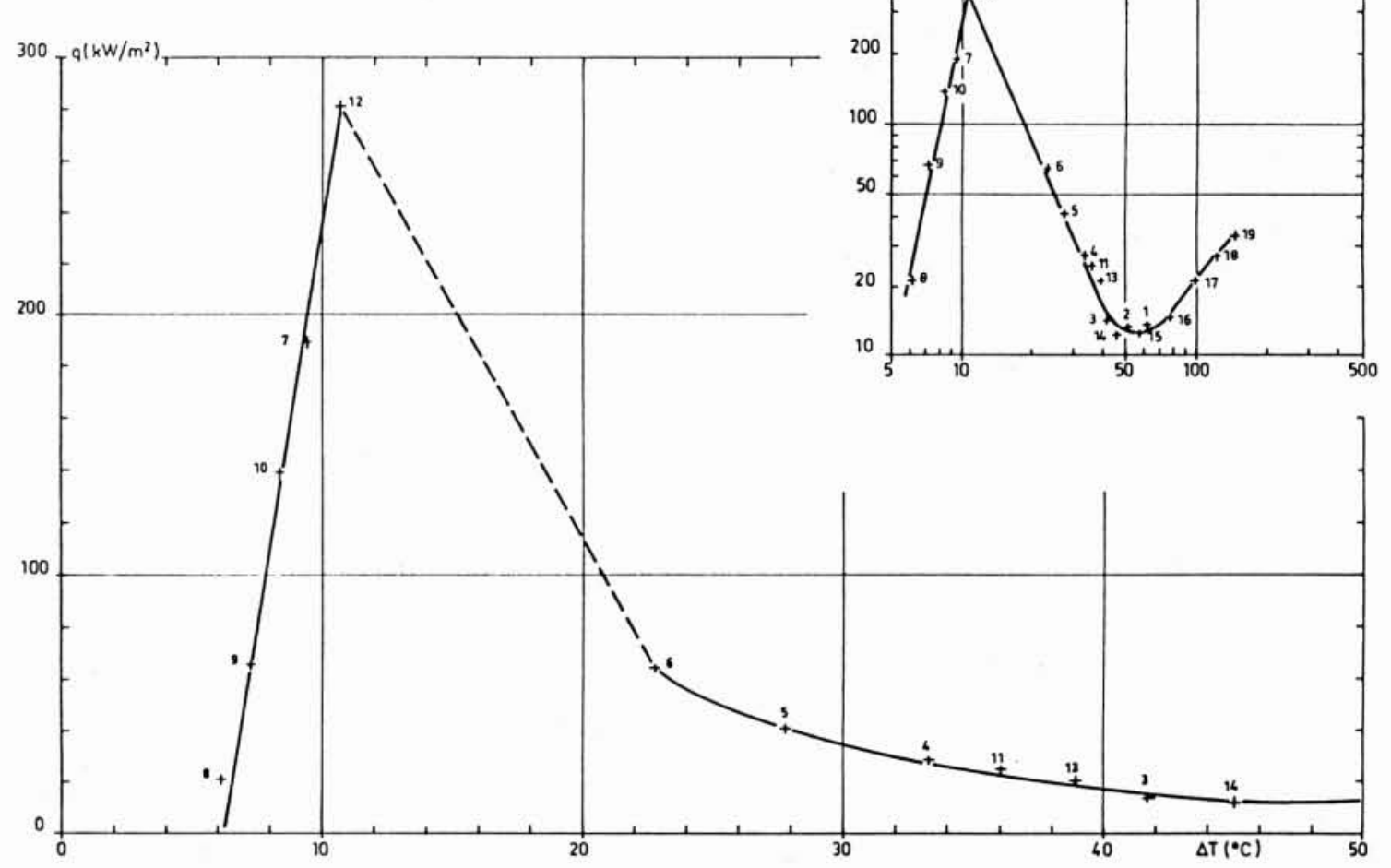

Figure 4. - Essais de Berenson (Cu-Pentane n) - Essai 10 (d'après [7]).

La courbe d'ébullition met en évidence deux points caractéristiques : un maximum $\left(q_{c}, \theta_{c}\right)$ correspondant aux conditions critiques et un minimum $\left(q_{m}, \theta_{m}\right)$ appelé le plus souvent point de Leidenfrost.

Lorsque le titre massique augmente, $q_{\mathrm{c}}$ diminue alors que $q_{m}$ varie relativement peu. Ceci explique qu'aux titres massiques "élevés" (voisins de 1) la courbe d'ébullition puisse devenir monotone croissante (figure 2).

La pente négative de la courbe d'ébullition, correspondant à la zone de transition (figure 3), a été mise en 
$\mathrm{q}\left(\mathrm{kW} / \mathrm{m}^{2}\right)$
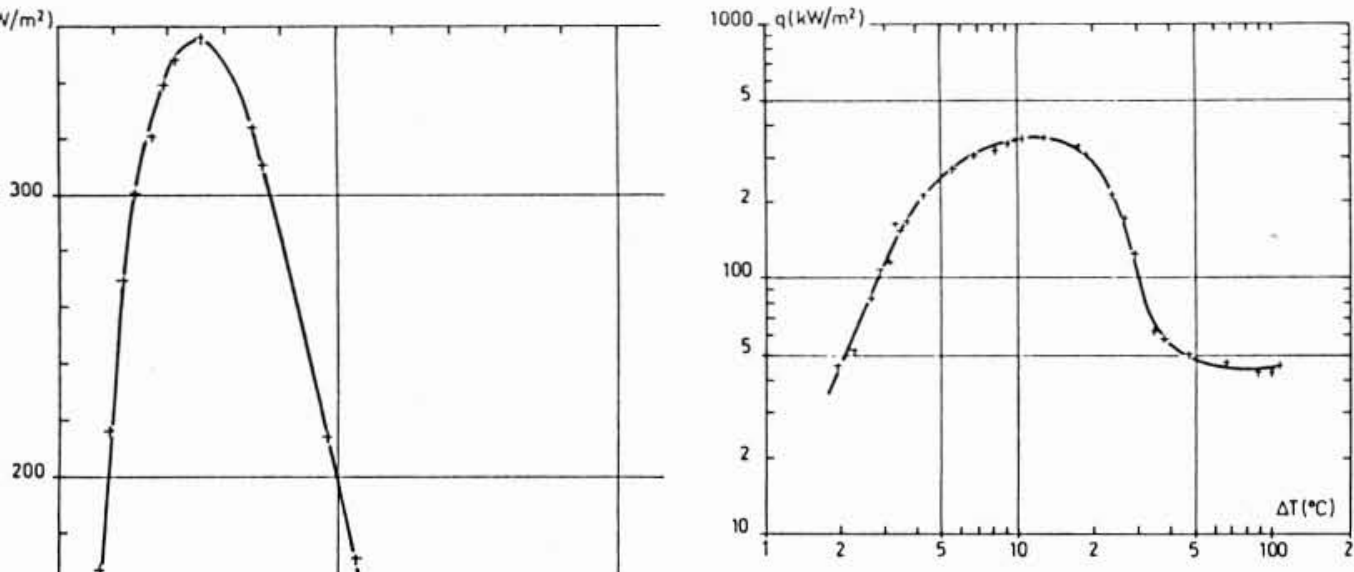

ssais de HESSE - R 12 - $P=14$ bor (D'après [9])

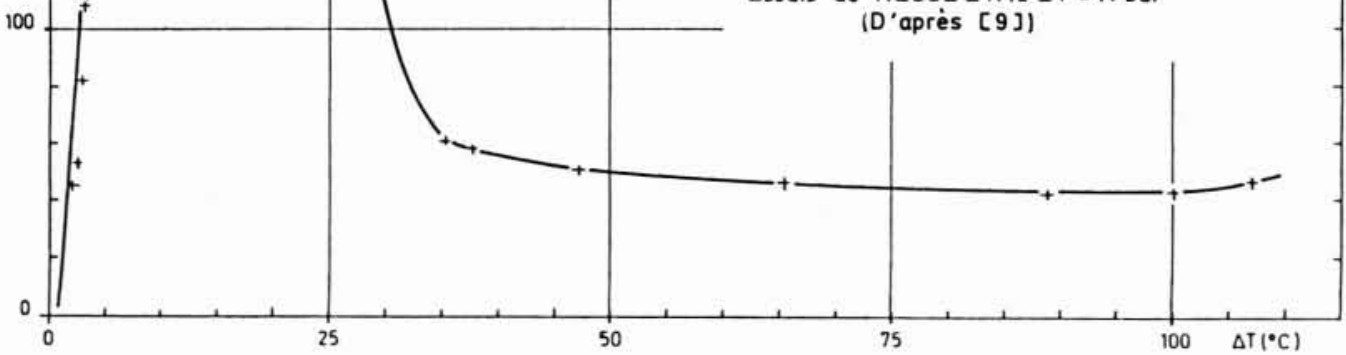

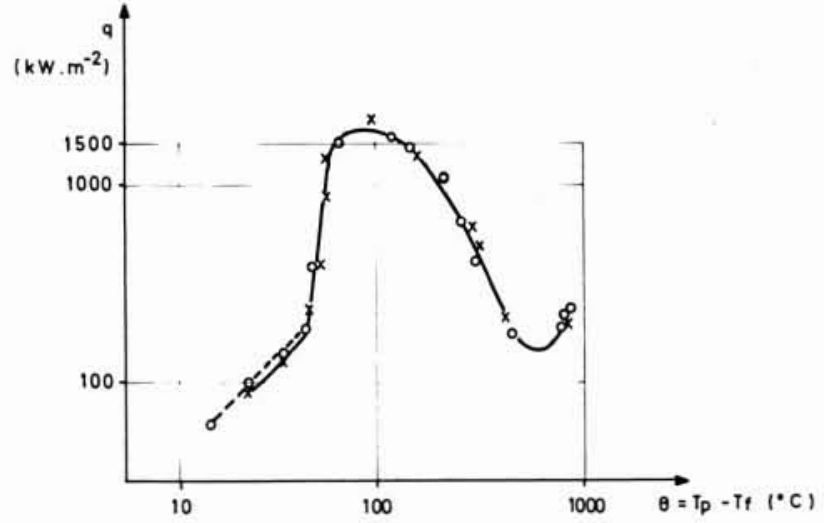

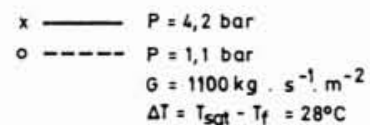

Figure 6. - Courbe d'ébullition de l'eau en régime sous-saturé. Résultats expérimentaux d'Ellion [10] (1954).
Figure 5

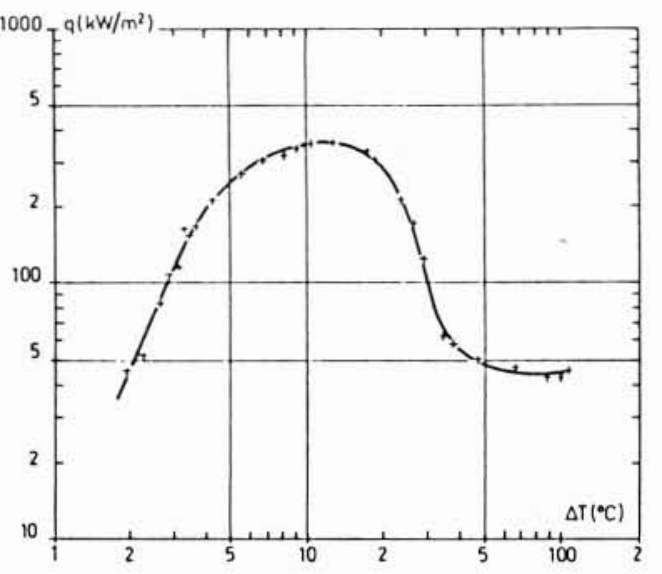

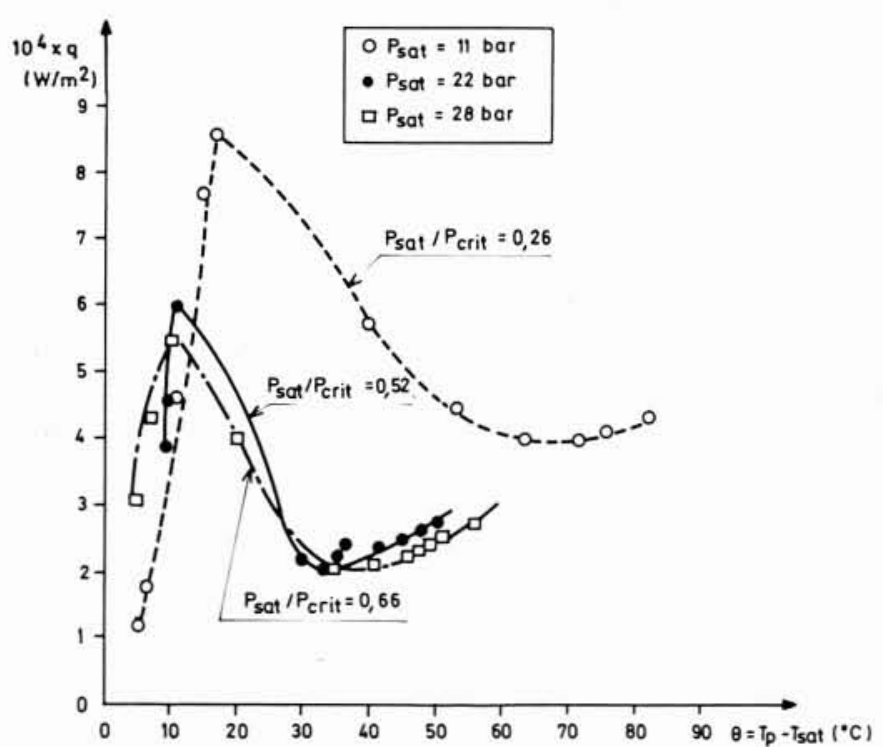

Figure 7. - Courbes d'ébullition du fréon en convection forcée. Influence de la pression $\left(\mathrm{G}=1350 \mathrm{~kg} / \mathrm{s} \cdot \mathrm{m}^{2}\right.$ - D'après Cumo et al. [13]. évidence, en ébullition stagnante, par NukiYama [6] en 1938, puis confirmée par de nombreuses expériences depuis, parmi lesquelles on peut citer celles de BERENSON [7] (figure 4), NisHikawA et al. [8] et HeSSE [9] (figure 5).

En convection forcée, la zone de transition à pente négative a été mise en évidence par ELLION (1954) [10] (figure 6) puis confirmée par un certain nombre d'expériences citées au paragraphe 1.4, et également par BLATT et ADt [11], Mc Donough et al. [12], Cumo et al. [13] (figure 7). 

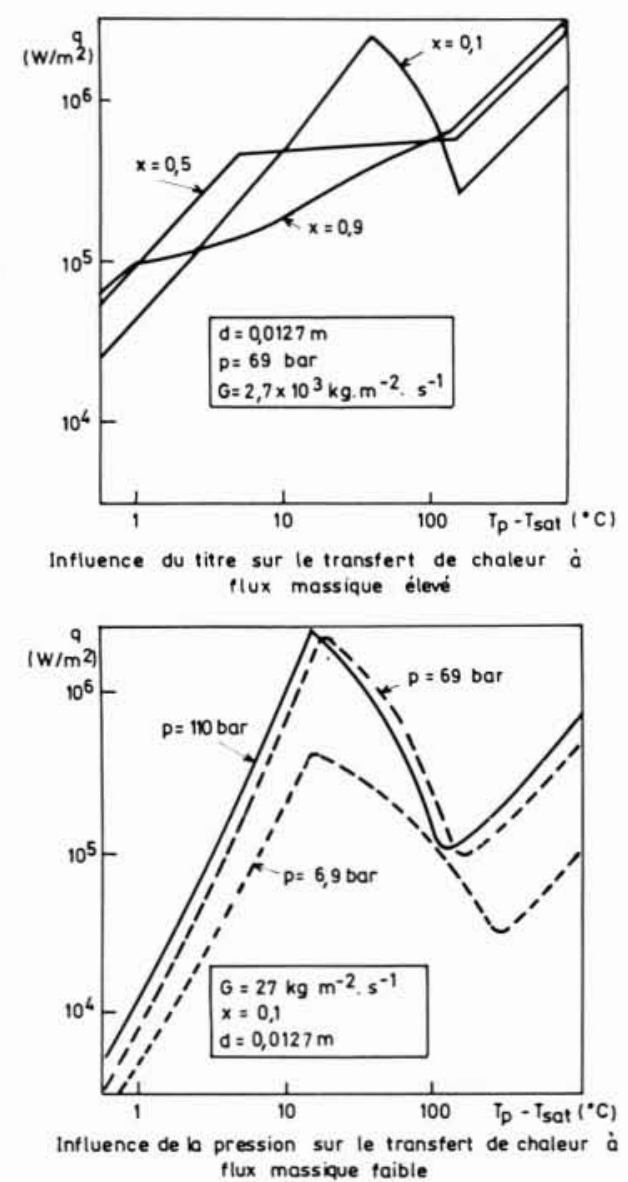

Figure 8. - Exemples de courbes d'ébullition reconstituées à partir de corrélations (D’après Bjornard et Griffith [18]). Nature de la paroi : couche d'oxyde sur acier inoxydable.

Cette zone de transition à pente négative tend, toutefois, à disparaitre et la courbe d'ébullition à devenir monotone croissante :

- lorsque la pression tend vers la pression critique thermodynamique. En ébullition stagnante, ce phénomène a été mis en évidence expérimentalement par DRAPER [14] et en ébullition en convection forcée, il est analysé par TOLUBINSKIY et al. [15] ;

- en convection forcée, lorsque le flux massique augmente. Des expériences, mettant en évidence ce phénomène, sont rapportées par FUKUYAMA et HIRATA [16] et par Groeneveld et Borodin [17]. TolubinskiY et al. analysent également ce phénomène en [15].

La figure 8 présente des exemples de courbes d'ébullition, "reconstituées" à partir des corrélations disponibles, relatives à chaque régime d'ébullition et aux conditions critiques et de LeIDENFROST [18]. Elles montrent l'influence respective du titre massique, de la pression et du flux massique sur ces courbes.

L'une des questions majeures qui se pose concerne la validité de l'hypothèse des conditions locales. Cette hypothèse est généralement celle adoptée dans les codes de calcul d'écoulements et de transferts thermiques diphasiques [18]. Il est clair que les conditions critiques ( $\left.q_{c}, x_{\varepsilon}\right)$, de même que les conditions minimales $\left(q_{m}, x_{m}\right)$, dépendent également des conditions amont.

A. - L'hypothèse des conditions locales a été notamment soigneusement examinée par BARNETT [5] dans le cas de tubes chauffés uniformément ou non. Pour des écoulements fortement sous-saturés, les conditions critiques dépendent uniquement des conditions locales. Pour des écoulements saturés, les effets de "l'histoire" du fluide, en amont, peuvent avoir une grande influence [19].

Dans le cas du chauffage uniforme, toutes choses égales, par ailleurs, la longueur chauffante amont a une influence d'autant plus faible que cette longueur est grande. Dans le cas du chauffage à densité de flux non uniforme, cette propriété a été également vérifiée par plusieurs auteurs [5].

La détermination expérimentale des conditions critiques montre que $q_{\mathrm{c}}$ devient indépendant de la longueur chauffante amont $\ell$ lorsque le rapport $\ell / d$ devient approximativement supérieur à 70 [20].

Des données relativement récentes, présentées par Shiralkar et al. [21], montrent que le point minimum peut être également fonction des conditions amont.

B. - Dans l'état actuel des connaissances, on peut supposer en résumé que les courbes d'ébullition « locales " sont valables lorsque la longueur chauffante amont est suffisante. Pour des tubes de faibles longueur, les courbes d'ébullition dépendent également de la longueur chauffante amont.

\subsection{Revue bibliographique}

On a identifié différents régimes d'ébullition tant en ébullition stagnante qu'en convection forcée. On a distingué principalement l'ébullition nucléée, l'ébullition de transition et l'ébullition en film. Le chauffage par effet Joule, à densité de flux thermique croissante, se caractérise par l'apparition de la crise d'ébullition lorsque le régime d'ébullition est l'ébullition nucléée, c'est-à-dire par une instabilité qui se traduit par l'augmentation, plus ou moins brutale, de la température de paroi. Son amplitude est d'autant plus grande que l'enthalpie locale du fluide est faible. Cette instabilité peut donc conduire, dans certaines conditions de fonctionnement défavorables, à la fusion de la paroi ou du moins à sa forte détérioration ( " burn-out "). On indiquera, au chapitre 2 , une interprétation simplifiée de ce mode d'instabilité.

Entre 1950 et 1970, l'essentiel des investigations expérimentales sur les écoulements diphasiques en ébullition concernaient l'ébullition nucléée et les conditions critiques. Ceci peut s'expliquer pour deux raisons qui ne sont pas indépendantes:

- d'une part, dans la plupart des systèmes en ébullition, on évite de dépasser les conditions d'ébullition critiques, du fait des risques d'apparition du "burn-out" et le régime d'ébullition le plus courant est l'ébullition nucléée. Il est, par contre, nécessaire de connaître avec précision ces conditions critiques;

- dans les systèmes expérimentaux, la même limitation existe, du fait qu'on utilise généralement des tubes chauffés électriquement. 
Il faut noter que, toutes choses égales par ailleurs, l'accroissement de température correspondant à la crise d'ébullition dépend de la nature du fluide. S'il est généralement élevé avec l'eau, il est plus modéré avec les fréons, relativement faible avec les fluides cryogéniques.

A partir des années 1970, l'intensification et l'approfondissement des études de sûreté des réacteurs nucléaires à eau conduisent à l'analyse détaillée de conditions accidentelles au cours desquelles apparaissent des phénomènes, des régimes d'ébullition, que l'on évitait soigneusement jusque là dans les systèmes à flux imposé : ébullition en film à faible titre et même à l'état sous-saturé (écoulement annulaire inversé). La simulation correcte des phénomènes transitoires impose, par ailleurs, une bonne connaissance de la zone d'ébullition de transition.

De même, dans les générateurs de vapeur à haut flux thermique (générateurs de vapeur chauffés au sodium, chaudières à rayonnement), la simulation des phénomènes transitoires requiert la connaissance des propriétés de transfert thermique dans des domaines de variation des paramètres thermohydrauliques (pression, flux massique, notamment) aussi larges que possible. La compréhension et la prédiction du phénomène local d'assèchement demandent une connaissance satisfaisante des lois de transfert de chaleur en ébullition en film et en ébullition de transition. Les connaissances sont malheureusement lacunaires pour deux raisons essentielles :

- l'inadaptation des dispositifs expérimentaux habituels, constitués par de longs tubes chauffés électriquement, qui ne permettent pas d'atteindre ou d'effectuer des mesures dans ces régimes d'ébullition et dans des domaines de variation des paramètres suffisamment larges;

- les résultats expérimentaux sont représentés sous forme de lois empiriques ou semi-empiriques (corrélations), caractéristiques d'un régime particulier d'ébullition ou d'une propriété particulière (conditions critiques notamment); il apparaît difficile, parfois impossible, dans l'état actuel des connaissances de représenter avec précision l'évolution globale du flux thermique lorsque, toutes choses égales par ailleurs, la température de paroi évolue localement.

Ces difficultés sont encore aggravées par l'influence importante que des paramètres "secondaires" peuvent avoir, comme l'état de surface de la paroi chauffante.

A partir de 1975 environ, la nécessité de simuler de grands transitoires dans les codes de calculs d'écoulements diphasiques " avancés " tels que Relap [22], Cathare [23], Trac [24] impose de disposer d'une bibliothèque élaborée de corrélations, permettant de calculer les propriétés de transfert thermique :

- dans une gamme très large de variation des paramètres thermohydrauliques. Par exemple : P de 150 à 1 bar, G de 5000 à $0 \mathrm{~kg} / \mathrm{s} \cdot \mathrm{m}^{2}$, en écoulement ascendant et, éventuellement, descendant;

- pour tous les régimes d'ébullition donc pour des écarts de température $\theta$ pouvant atteindre $1200^{\circ} \mathrm{C}$.

La notion de courbe d'ébullition, étendue de l'ébullition stagnante à l'ébullition en convection forcée, tend à se généraliser. Dans Relap, elle est nommée : technique de la surface de transfert de chaleur [22]. Cette notion est utilisée, non seulement pour élaborer la "bibliothèque " évoquée ci-dessus, mais également pour tenter d'interpré-

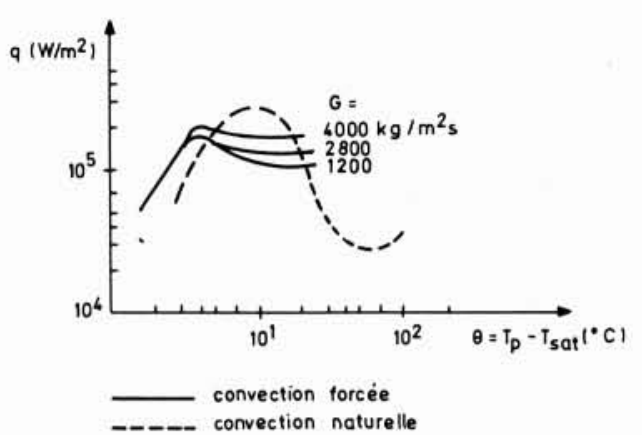

Figure 9. - Courbes d'ébullition du fréon 114 en convection naturelle et en convection forcée. Influence du flux massique $\left(\mathrm{P}_{\mathrm{sat}}=12\right.$ bar $)-$ D'après Stephan et Hoffmann [26].

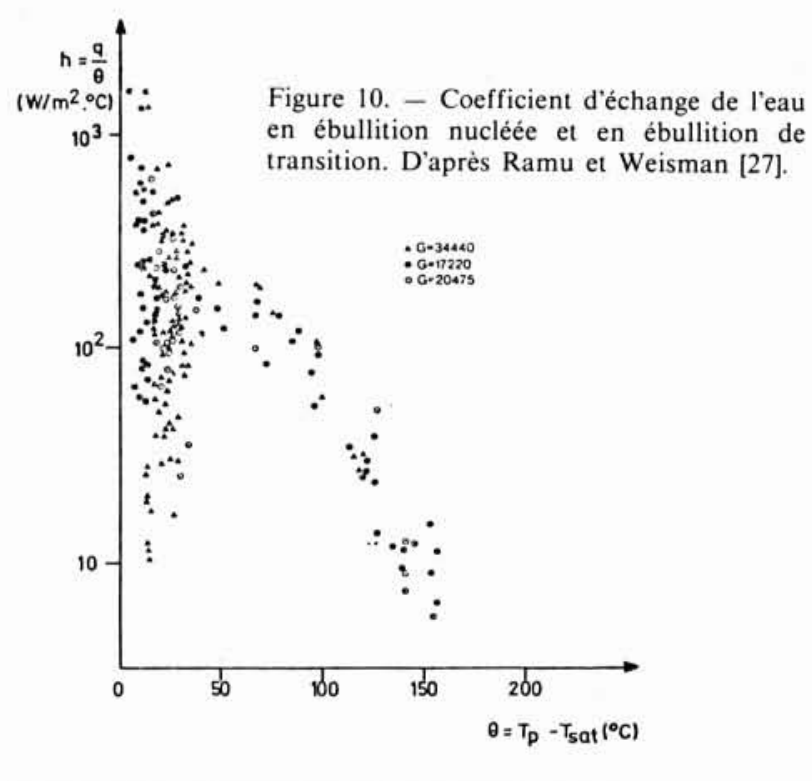

ter qualitativement les phénomènes survenant au cours des régimes transitoires [25].

\subsection{Recherches expérimentales concernant les courbes d'ébullition}

Parallèlement, un certain nombre de laboratoires étrangers développent des procédures expérimentales pour permettre l'obtention globale de ces courbes d'ébullition : c'est le cas des travaux du MIT et de l'Université de Cincinnati, aux Etats-Unis, de l'AECL, au Canada, de l'UKAEA en Grande-Bretagne.

Deux techniques principales sont utilisées :

1) Utilisation d'un fluide chauffant, afin de tenter d'obtenir un système à température imposée. Les expériences s'effectuent en régime stationnaire. L'augmentation de la densité de flux thermique est provoquée par l'augmentation de la température d'entrée du fluide chauffant. Les expériences de STEPHAN et Hoffman [26] (figure 8), de RAMU et WEISMAN [27] (figure 10) et de YILMAZ [28] appartiennent à cette catégorie. ELLION [10] (figure 6) peut être considéré comme le précurseur de cette technique. 
2) Utilisation d'une cellule chauffante à forte capacité calorifique que l'on chauffe au-delà des conditions critiques correspondant à l'essai envisagé, sans présence du liquide bouillant. Le liquide en ébullition est ensuite dérivé à travers la cellule dans laquelle on a coupé la puissance électrique de chauffage. L'évolution en régime transitoire de la température de paroi de la cellule permet de reconstituer une courbe d'ébullition. L'essai s'effectue en assurant la constance des conditions thermohydrauliques à l'entrée de la cellule (pression, flux massique, enthalpie). Les expériences de Groeneveld [29], Cheng [30], du Mit [31] et des laboratoires d'Harwell [32] appartiennent à cette catégorie.

Ces deux méthodes expérimentales présentent des inconvénients :

1) Dans le cas des expériences à température de paroi imposée, la détermination du flux de chaleur local est plus imprécise que dans des expériences utilisant le chauffage électrique. Celle-ci s'effectue à partir de la mesure d'écarts de température, généralement faibles, dans le fluide chauffant. En outre, pour atteindre certaines températures de paroi élevées, il faut pouvoir disposer d'un fluide chauffant pouvant, lui-même, atteindre des températures élevées. Si on étudie les propriétés de transfert thermique de l'eau, on est pratiquement conduit à l'utilisation de fluides chauffants comme les métaux liquides (sodium, notamment [33]).

Si l'on veut, par ailleurs, garantir des conditions thermiquement stables comme on le verra au chapitre 2 , il faut pouvoir assurer un coefficient d'échange thermique du fluide chauffant élevé et une épaisseur de paroi faible, ce qui peut être incompatible avec des contraintes de sécurité et de tenue mécanique des tubes. Ramu et WeISMaN utilisent, par exemple, du mercure. La dispersion des résultats est relativement forte [27] (figure 10).

2) Les expériences du deuxième type sont conduites en régime transitoire. Certaines imprécisions sont entraînées par la rapidité du passage de la zone de transition et les effets liés à la conduction axiale, c'est-à-dire à la propagation du front de remouillage le long du tube [33], [34]. Par ailleurs, on n'est pas assuré que le trajet, suivi localement, par un élément de la surface chauffante, dans le plan $(q, \theta)$ au cours du régime transitoire est celui correspondant à la courbe d'ébullition en régime stationnaire. En général, le point de retour au régime d'ébullition nucléée, soit $M$ sur la figure 1 , correspond à environ $80 \%$ de la valeur au point maximum $C$ [36].

\subsection{Autre méthode de recherche des courbes d'ébulli- tion : la stabilisation électronique}

Une autre technique, développée à la Direction des Etudes et Recherches de l'EDF et à l'ENSTA, consiste à garantir la stabilité du processus d'ébullition en utilisant un système de chauffage électrique asservi. L'intensité du courant circule dans une cellule d'essai dont les dimensions sont analogues à celles des expériences du $2^{c}$ type mentionnées ci-dessus mais de faible épaisseur, donc à faible capacité thermique. L'intensité du courant est asservie à une grandeur représentative de la température de paroi : soit une mesure directe, locale, de la température, soit une grandeur moyenne, notamment, par la mesure de la résistance électrique de la cellule [37], [38].

Historiquement, ce dispositif a été d'abord développé par Peterson et ZAALOUK avec des fils de platine chauffants, en ébullition stagnante (1962-1966) [39] puis en convection forcée [40]. Des dispositifs du même type ont été développés en URSS par Kovalev [41] et au Japon par SAKURAI et al. [42] pour l'ébullition stagnante.

L'originalité de la démarche développée par EDF et l'ENSTA consiste à utiliser les principes de la cellule d'essai utilisée dans les expériences de remouillage en régime transitoire et de la régulation de l'intensité du courant. Aucune expérimentation systématique n'avait été entreprise en utilisant ces deux principes, c'est-à-dire en assurant :

- des mesures en régime stationnaire:

- une surface chauffante géométriquement représentative (tube) de celles généralement utilisées industriellement;

- une exploration systématique de l'influence des divers paramètres thermohydrauliques sur l'allure de la courbe d'ébullition.

La démarche suivie dans la mise au point de ce dispositif de stabilisation a été progressive; des essais ont été réalisés successivement :

- avec un fil platine, en eau, en ébullition stagnante [37]; - avant un fil de platine placé dans un tube parcouru par un écoulement de fréon 113 [43];

- avec un tube, refroidi par un écoulement de fréon 113 [38], [44].

On rappelle, ci-dessous, le principe complémentaire sur lequel repose ces expériences: le concept de stabilité thermique. On présente dans une seconde partie (à paraître dans un prochain numéro), quelques résultats marquants obtenus avec ce dispositif. Les résultats sont comparés à ceux connus habituellement dans la littérature.

Figure 11. - Schéma fonctionnel d'un élément cylindrique chauffant stabilisé électroniquement.

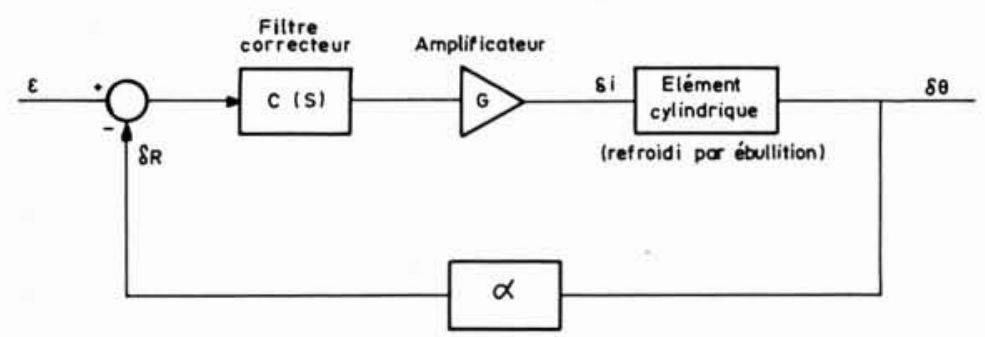




\section{Le concept de stabilité thermique}

Un certain nombre d'auteurs, depuis les années 1960, ont introduit et développé cette notion essentielle de stabilité thermique, pour la compréhension du comportement thermique macroscopique, global ou local, d'une surface chauffante refroidie par un liquide en ébullition. ELLION [10], puis ADIUTORI [45] ont posé ce concept de façon intuitive. Stephan [46] et Kovalev [47], notamment, ont développé ce concept. Llory et PLANChard en ont présenté également une approche [48].

Afin de présenter cette notion, on considère un système très simplifié comportant un tube de diamètre $d$ et de courte longueur, dans lequel on assure une circulation de liquide en ébullition à la température constante $T_{f}$. On néglige les distributions longitudinales et radiales de température dans le tube. On appelle système interne le fluide bouillant et système externe la source de chaleur assurant le chauffage. Celle-ci peut être d'origine électrique ou constituée d'un fluide en convection forcée circulant sur la paroi externe du tube.

Le flux, engendré par le système externe à la paroi interne, est supposé ne dépendre que de l'écart de température entre la paroi interne et le liquide bouillant, soit $\theta=T_{r}-T_{r}$.

\subsection{Systèmes en "boucle ouverte"}

Globalement, la paroi est un volume caractérisé par une seule température "lumped parameter system "; elle est régie par l'équation de conservation :

$\rho_{m} C_{m} \frac{d T_{p}}{d t}=\frac{1}{D}\left(q_{e}-q_{i}\right)$

où $D$ est une dimension caractéristique :

$$
D=\frac{\text { volume de la paroi }}{\text { surface d'échange }}
$$

Si on pose $\theta=T_{p}-T_{f}\left(\right.$ si $\left.T_{f}=\mathrm{Cte}\right)$ :

$$
\rho_{m} C_{m} \frac{d \theta}{d t}=\frac{1}{D}\left(q_{c}-q_{i}\right)
$$

En régime stationnaire, on a évidemment $q_{e}=q_{i}=q_{0}$ pour $\theta=\theta_{0}$. En écrivant l'équation aux petites perturbations, il vient :

$$
D \rho_{m} C_{m} \frac{d \delta \theta}{d t}=\left(\frac{\partial q_{e}}{\partial \theta}-\frac{\partial q_{i}}{\partial \theta}\right) \cdot \delta \theta
$$

avec :

$$
\delta \theta=\theta(t)-\theta_{0} .
$$

Le système est stable si et seulement si :

$$
\frac{\partial q_{c}}{\partial \theta}<\frac{\partial q_{i}}{\partial \theta}
$$

Pour de tels systèmes définis comme ci-dessus, on peut déduire de ce critère de stabilité quelques conclusions intéressantes :
1) Le terme $\partial q_{r} / \partial \theta$ est, en général, négatif ou nul. Donc si $\partial q_{i} / \partial \theta>0$, ce qui est généralement le cas en convection forcée et toujours le cas en écoulement monophasique subcritique, la stabilité est toujours assurée. C'est donc également le cas en ébullition en convection forcée, à titre élevé ou lorsque la pression ou le flux massique sont èlevés.

2) En ébullition, dans le cas de l'existence de la zone de transition, pour laquelle $\partial q_{i} / \partial \theta<0$, on peut distinguer deux cas principaux :

2.1) $\mathrm{Si} \partial q_{c} / \partial \theta=0$ (chauffage électrique, résistance électrique indépendante de la température) : le critère de stabilité devient $\partial q_{i} / \partial \theta>0$. Ce cas correspond à un système à flux imposé. Il n'est donc, en général, pas possible d'obtenir la zone de transition avec un système chauffé électriquement, à intensité imposée.

Si la résistance électrique dépend de la température, ce qui est généralement le cas, à intensité du courant imposée, on peut écrire :

$$
\frac{\partial q_{e}}{\partial \theta}=\frac{i^{2}}{A} \frac{d R}{d \theta}
$$

Comme $d R / d \theta$ est généralement positif pour les métaux, ce cas est encore plus défavorable sur le plan de la stabilité thermique que le cas d'un système à "flux imposé ".

2.2) Avec un système de chauffage utilisant un autre fluide, on peut écrire en première approximation :

$$
q_{c}=h\left(T_{\mathrm{c}}-T_{p}\right)
$$

où $h$ est le coefficient d'échange global entre le fluide chauffant à la température $T_{\mathrm{r}}$ et la paroi en contact avec le liquide bouillant à la température $T_{0}$; pour une paroi cylindrique :

$$
\frac{1}{h}=\frac{1}{h_{0}}+\frac{1}{\left[d_{i} \log \frac{d_{e}}{d_{i}}\right]}
$$

On peut écrire :

$$
q_{e}=h\left(T_{c}-T_{0}\right)-h \theta, \text { soit } \frac{\partial q_{e}}{\partial \theta}=-h .
$$

Dans ces conditions, le système peut être stable ou instable suivant les valeurs respectives de $\partial q_{i} / \partial \theta$ et de $h$ donc, en particulier, de la vitesse du fluide chauffant et de l'épaisseur et de la nature de la paroi choisie.

On voit, en particulier, que même dans le cas où $h_{0}$ est très grand, il existe une épaisseur maximale $e$ pour ne pas risquer d'instabilité thermique dans le cas d'une paroi mince :

$$
\frac{\partial q_{i}}{\partial \theta}=-\frac{\lambda}{e} \quad e<\frac{\lambda}{\left|\frac{\partial q_{i}}{\partial \theta}\right|}
$$


Ainsi, en ébullition stagnante :

- les expériences de BERENSON ont été faites dans des conditions instables comme l'ont montré différents auteurs : AdIUTORI [49], CANON et PARK [50], ce que ne permet pas de mettre en évidence la représentation logarithmique $q(\theta)$ adoptée par BERENSON mais est nettement mis en évidence par la représentation des mêmes courbes en coordonnées linéaires (cf. figure 4):

- les expériences de Hesse [9] ont été effectuées de façon stable, le dispositif expérimental ayant été conçu a priori pour satisfaire le critère de stabilité thermique (figure 5).

En ébullition en convection forcée, ELLION [10] ( $f$ gure 6) a posé de façon intuitive le critère de stabilité thermique et a réalisé son dispositif expérimental pour satisfaire ce critère. Depuis ces expériences, peu d'auteurs, utilisant un fluide chauffant comme source de chaleur, mentionnent ce critère de stabilité et s'assurent s'il est vérifié. Le seul exemple à notre connaissance est celui de Stephan et Hoffmann [26] (figure 9).

\subsection{Systèmes en "boucle fermée "}

Les systèmes précédents sont supposés en boucle ouverte. $\mathrm{Si}$ on reprend le même problème de stabilité, avec les mêmes hypothèses générales mais en supposant que l'intensité $i$ est asservie à la mesure de la température de paroi (figure 11), on peut écrire :

$$
\frac{\delta \theta(s)}{\delta q_{e}(s)}=\frac{A K_{0}}{s+B_{0}} \text { avec : } K_{0}=\frac{1}{\rho_{m} C_{m} V} \text { et } B_{0}=K_{0} \frac{\partial q_{i}}{\partial \theta}(4)
$$
$q_{e}$ est par ailleurs de la forme $R i^{2} / A$, soit :

$$
\delta q_{e}=\frac{\mathrm{i}^{2}}{A} \delta R+\frac{2 R i}{A} \delta i
$$

En supposant que la résistance électrique de la paroi chauffante varie linéairement avec la température, soit : $\delta R=\alpha R_{0} \delta \theta$, il vient :

$$
A \cdot \frac{\delta q_{c}(s)}{\delta i(s)}=i_{0}^{2} \alpha R_{0} \frac{\delta \theta}{\delta i}+2 R_{0} i_{0}
$$

En combinant les relations (4) et (5) :

$$
\frac{\delta \theta(s)}{\delta i(s)}=\frac{2 R_{0} i_{0} K_{0}}{s+B_{0}-K_{0} i_{0}^{2} \alpha R_{0}}=\frac{K}{s+B}
$$

Il est clair que dans la zone d'ébullition de transition, pour laquelle $B_{0}<0$, le système en boucle ouverte est instable.

Si on suppose l'existence d'un filtre correcteur proportionnel (figure 10), dont la fonction de transfert est par conséquent : $C(s)=C=\mathrm{Cte}$, on peut écrire :

$$
G C(s)[\varepsilon(s)-\delta R(s)]=\delta i
$$

Soit en utilisant la relation (6) :

$$
\frac{\delta \theta(s)}{\varepsilon(s)}=\frac{K G C(s)}{s+B+\alpha K G C(s)}
$$

La condition de stabilité du système impose qu'il n'y ait pas de pôle à partir réelle positive, soit : ou, en tenant compte de $B$ :

$$
C>\frac{\mathrm{K}_{0} i_{0}^{2} \alpha R_{0}-B_{0}}{\alpha K G}
$$

Cette théorie simplifiée montre qu'il est possible de stabiliser dynamiquement un système thermiquement instable.

\subsection{Remarque importante}

Les exemples traités dans les deux paragraphes précédents sont évidemment schématiques. Une théorie plus complète devrait tenir compte de la distribution axiale de température de la paroi chauffante, et des conditions aux limites aux bornes de l'élément cylindrique chauffant. Il est par conséquent nécessaire de tenir compte de l'influence de la conduction axiale [51] et de plus, en convection forcée, de l'évolution du titre massique le long de la paroi chauffante. Le problème est encore plus difficile lorsque la source de chaleur est constituée par un autre fluide; il existe évidemment un couplage thermique entre les deux fluides, chauffant et bouillant.

Le problème de la stabilité thermique devient donc beaucoup plus complexe, et il est nécessaire de faire appel à des méthodes de résolution numériques. Des études récentes ont montré que la méthode de continuation était particulièrement bien adaptée à l'étude du comportement thermique de surfaces chauffantes refroidies par ébullition [52], [53].

Toutefois, le critère (3) constitue une condition suffisante de stabilité thermique et d'unicité de l'état stationnaire.

\section{Conclusion}

On a essayé de mettre en évidence l'intérêt de la notion de courbe d'ébullition pour représenter globalement les propriétés de transfert thermique entre une paroi chauffante et un écoulement diphasique en ébullition. Cette notion tend à se généraliser dans la littérature spécialisée et des expériences spécifiques sont développées depuis 1975 principalement pour obtenir ces courbes d'ébullition dans leur totalité c'est-à-dire y compris la zone de transition.

On a également présenté la notion de stabilité thermique qui doit être utilisée conjointement à celle de courbe d'ébullition.

Dans un second article, on examinera de façon détaillée des courbes d'ébullition expérimentales obtenues par divers auteurs et l'on comparera celles-ci aux courbes d'ébullition "reconstituées" à partir des corrélations disponibles.

$$
B+\alpha K G C>0
$$




\section{Références bibliographiques}

[1] W.R. GambiLl. - Burnout in boiling heat transfer. Part I: Pool-boiling systems. Nuclear Safety, vol. 9, no 5, septembreoctobre 1968, p. 351-362.

[2] A.E. Bergles. - Burnout in boiling heat transfer. Part I : pool-boiling systems. Nuclear Safety, vol. 16, n 1, JanuaryFebruary 1975.

[3] A.E. Bergles. - Pool boiling. In : Two-Phase Flow and Heat transfer in the Power and Process Industries (A.E. BERGLESJ.C. Collier - J.M. Del.haye - G.F. Hewitt - F. MAYINGer). Hemisphere Publishing Corp., 1981, p. 190-225.

[4] S.W. GouSE Jr. - An index to the two-phase gas-liquid flow literature. The MIT Press, Cambridge, Massachusetts (USA), 1966.

[5] R.V. MACBETH. - The burn-out phenomenon in forcedconvection boiling. Advances in Chemical Engineering, vol. 7, 1968, p. 207-293.

[6] S. Nukiyama. - The maximum and minimum value of the heat $\mathrm{Q}$ transmitted from metal to boiling water under atmospheric pressure. J. Japan Soc. of Mechanical Engineers, vol. 37, 1934, p. 367-374. Traduction en Anglais : Int. J. Heat Mass Transfer, vol. 9, 1966, p. 1419-1433.

[7] P.J. Berenson. - Experiments on pool boiling heat transfer. Int. J. Heat Mass Transfer, vol. 5, 1962, p. 985-999.

[8] K. Nishikawa, S. Hasegawa, H. Honda. - Studies on boiling characteristic curve. Memoirs of the Faculty of Engng Kyushu Univ., vol. XXVII, no 3, Fukuoka, Japan, 1967, p. 133-154

[9] G. HESSE. - Heat transfer in nucleate boiling, maximum heat flux and transition boiling. Int. J. Heat Mass Transfer. vol. 16, 1973, p. 1611-1627.

[10] M.E. ELLion. - A study of the mechanism of boiling heat transfer. Memorandum no 20-88, Jet Propulsion Laboratory, California Institute of Technology, $1^{\text {er }}$ mars 1954

[11] J.A. BLATT, RR. ADT Jr. - An experimental investigation of boiling heat transfer and pressure-drop characteristics of Freon 11 and Freon 113 refrigerants. A.I.ch.E. Journal, vol. 10 , no 3, May 1964, p. 369-373.

[12] J.B. Mc Donough, W. MiLich, E.C. King. - An experimental study of partial film boiling region with water at elevated pressures in a round vertical tube. Chemical Eng. Progress Symp. Series, vol. 57, no 32, 1961, p. 197-208, 4th Nat. Heat Transfer Conf. Buffalo, August, 14-17, 1960.

[13] M. Cumo, G.E. Farello, G. Palazzi. - "Temperature controlled" and "heat flux controlled" systems. La Termotecméca, vol. XXXI, n॰ 6, 1977, p. 313-321

[14] R. DRAPER. - Natural convection and boiling heat transfer with $S F_{0}$ in the région of the critical point. Thèse Univ. De Manchester (G.B.). Dep. of Nuclear Engineering. $1^{\text {er }}$ octobre 1967.
[15] V.I. TolubinskiY, A.A. VASIL'Yev, A.A. Mitin. - Temperature of the pipe wall in transition from nucleate to film boiling. Heat Transfer Soviet Research, vol. 15, no 1, January-February 1983, p. 1-6.

[16] Y. Fukuyama, M. HiRata. - Boiling heat transfer characteristics with high mass flux and disappearance of $\mathrm{CHF}$ following to DNB. 7th Int. Heat Transfer Conf. Munich, 1982 (September 6-10), vol. 4, Paper FB17, p. 273-278.

[17] D.C. Groeneveld, A.S. Borodin. - Occurrence of slow dryout in forced convective flow. Congrès CERI - Miami Beach (USA), 16-18 avril 1979.

[18] T.A. BJORNARD. - Blowdown heat transfer in a pressurized water reactor. Thèse M.I.T., août 1977.

[19] T.G. TheOFAnOus. - The boiling crisis in nuclear reactor. Safety and performance. Int. J. of Multiphase Flow; vol. 6, 1980, p. $69-95$.

[20] J.G. Collier. - Convective boiling and condensation. Seconde Edition, 1981, Mac-Graw Hill Book.

[21] B.S. Shiralkar, R.A. Hein, G. Yadigaroglu. - The "boiling curve" in high quality flow boiling. Proc. of the ANS/ASME/NRC Int. Topical Meeting on Nuclear Reactor Thermal Hydraulics. NUREG/CP-0014, vol. 2, Saratoga Springs, New-York, 1980.

[22] RELAP/MOD1 code manual, vol. 1: system models and numerical methods. - W.H. RANSON, R.J. WAGNER, J.A. Trapp, K.E. Carlson, D.M. Kiser, H. Hauo, H. Chow, R.A NELSON, S.W. JAMES. NUREG/CR-1826-EGG-2070 (EG and G Idaho), March 1982.

[23] Equipe Mixte EDF-CEA-FRAMATOME: - CATHARE, Code CATHARE 1. Dossier descriptif D 235-1. Note de synthèse sur les transferts d'énergie en paroi, août 1983.

[24] TRAC-PF1: An advanced best estimate computer program for pressurized water reactor analysis. NUREG/CR-3567 LA-9944 MS - February 1984.

[25] R.A. NELSON. - Forced-convective post-CHF heat transfer and quenching. Journal of Heat Transfer, Trans. ASME, vol. 104, February 1982, p. 48-54.

[26] K. Stephan, E.G. HoffmanN. - Transition and flow boiling heat transfer inside a horizontal tube. Int. J. Heat Mass Transfer, vol. 20, 1977, p. 1381-1387.

[27] K. Ramu, J. Weisman. - Transition flow boiling heat transfer to water in a vertical annulus. Nuclear Eng. and Design, vol. 40, 1977, p. $285-295$.

[28] S. Yilmaz, J.W. Westwater. - Effect of velocity on heat transfer to boiling freon 113. Trans. of the ASME. J. of Heat Transfer, vol. 102, February 1980, p. 26-31.

[29] K.K. FUNG. - Forced convective transition boiling. Thèse. Université de Toronto, Dep. of Mechanical Eng., 1976. 
[30] S.C. Cheng, W.W.L. NG, K.T. Heng. - Measurements of boiling curves of subcooled water under forced convective conditions. Int. J. Heat Mass Transfer, vol. 21, 1978, p. 1385-1392.

[31] D.N. Plummer, O.C. Iloeje, P. Griffith, N.M. Rohsenow. - A study of post critical heat flux heat transfer in a forced convection system. M.I.T. Dep. of Mechanical Eng., Report no DSR 73645-80. March 1973.

[32] F.J. NEWBOLD, J.C. RALPH, J.A. WARD. - Post-dryout heat transfer under low flow and low quality conditions. AERE Harwell (G.B.), AERE-R8390, October 1976.

[33] D.M. FrancE. - DNB in liquid metal heated forced convection boiling. Int. J. Heat Mass Transfer, vol. 16, 1973, p. 2343-2354.

[34] P. Chambre, E. EliAs. - Rewetting model using a generalized boiling curve. Rapport EPRI, NP-571, octobre 1977.

[35] O.C. Illoeje, D.N. Plummer, W.M. Rohsenow, R. Griffith. - An investigation of the collapse and surface rewet in film boiling in forced vertical flow. J. of Heat Transfer. Trans. ASME, mai 1975, p. 166-172.

[36] J.G. Col.tier. - Heat transfer in the post burnout region and during quenching and reflooding (p. 6-142 à 6-188). Handbook of Multiphase Systems. Mac Graw Hill, G. HetSRONI Editeur, chapitre 6.-5, 1982.

[37] R. BENEJEAN, M. LlORY. - Stabilisation électronique d'un processus thermique non linéaire. EDF. Bulletin de la Direction des Etudes et Recherches, Série A, no 3, 1981, p. 43-50.

[38] D. GentILE. - Etude expérimentale de l'ébullition du Fréon 113 en convection forcée, à l'aide d'un dispositif électronique de stabilisation. Rapport final du contrat EDF-DER-RNETTA, T3IL04-2E $6609,1984$.

[39] W.C. Peterson, M.G. ZaAlouk. - Boiling curve measurements from a controlled heat transfer process. Journal of Heat Transfer. Trans. ASME, novembre 1971, p. 408-412.

[40] W.C. Peterson, M.M. Abdul Fetouh, M.G. Zaalouk. Boiling curve measurements from a controlled forced convection process. Conf. On Boiler Dynamics and Control in Nuclear Power Stations - BNES Conf., Londres, Mars 1973, p. $18-1$ à $18-6$, p. G6.

[41] S.A. Zhukov, V.V. BARELKo. - Nonuniform steady states of the boiling process in the transition region between the nucleate and film regimes. Int. J. Heat Mass Transfer, vol. 26, no 8: p. $1121-1130$.
[42] A. Sakural, M. Shiotsu. - Temperature-controlled poolboiling heat transfer. Proc. 5th Int. Heat Transfer Conf., Tokyo, 1974, Paper B3-1, p. 81-85. $5^{\prime}$ Conf. Int. sur le Transfert de chaleur. Tokyo, 1974, communication B3-1, p. 81-85.

[43] M. HADJ-SHEIKH. - Etude du processus de transfert de chaleur en ébullition en convection forcée. Thèse de DocteurIngénieur, ENSTA, juin 1982.

[44] D. Gentile, M. Llory, R. Benejean. - Stabilization of Freon 113 subcooled boiling in forced convection. Int. Communications Heat Mass Transfer. A paraître, 1985.

[45] E.F. ADIUTORI. - New theory of thermal stability in boiling systems. Nucleonics, Vol. 22, no 5, mai 1964, p. 92-101.

[46] K. Stephan. - Stabilität bein sieden (en allemand). $B W K$, vol. 17, no 12, December 1965, p. 571-578. Trad. EDF-DER n。 $1780 \mathrm{CH}$ (Stabilité de l'ébullition).

[47] S.A. Kovalev, - The stability of types of boiling. Teplofizika Wisokikh Temperatur, vol. 2, no 5, septembre-octobre 1964, p. 780-788 (traduction en anglais, p. 703-709).

[48] M. Llory, J. Planchard. - Introduction au concept de stabilité thermique : expression du problème général. $E D F$, Bulletin de la Direction des Etudes et recherches, série A, n० 3 , 1981, p. $35-42$.

[49] E.F. Adiutorı. - The New Heat Transfer. Vol. 1, Ventuno Press - Cincinatti (USA).

[50] R.M. CANON, E.L. PARK Jr. - Transition boiling of normal pentane from a horizontal flat gold surface at one atmosphere pressure. Int. J. Heat Mass Transfer, vol. 19, 1976, p. 696-698.

[51] G. JOLY, J.P. KeRNEVEZ, M. LLORY. - Thermal instability in pool boiling on wires at constant pressure. SIAM J. of Applied Mathematical, vol. 43, no 6, décembre 1983, p. 1294-1309.

[52] G. JoLY. - Analyse des solutions multiples dans les systèmes distribués. Thèse d'Etat. Université de Technologie de Compiègne, 1982.

[53] O. DE BAYSER. - Amélioration d'une méthode de cheminement par collocation et maillage adaptatif. Application à des problèmes d'instabilité thermique. Thèse de Docteur-Ingénieur. Université de Technologie de Compiègne. Décembre 1984.

[54] A. Era, G.P. Gaspari, A. Hassid, A. Milani, R. Zavatta. RELLI. - Heat transfer data in the liquid deficient region for steam-water mixtures at $70 \mathrm{~kg} / \mathrm{cm}^{2}$ flowing in tubular and annular conduits. Rapport du CISE-R-184, Topical report n० 11, juin 1966. 